\title{
OSCILLATING ANTIFERROMAGNETISM OF ULTRATHIN EuTe LAYERS*
}

\author{
Z. WILAMOWSKI \\ Institute of Physics, Polish Academy of Sciences \\ Al. Lotników 32/46, 02-668 Warszawa, Poland \\ G. SPRingholz, V. SVRCEK and W. JantsciI \\ Johannes Kepler Universität, 4040 Linz, Austria
}

\begin{abstract}
We study magnetic resonance on EuTe/PbTe superlattices. Analysis of the magnetic dipole anisotropy in the superlattices and of the EPR amplitude of isolated $\mathrm{Eu}$ ions in the PbTe wells shows that the diffusion at interfaces is very small. The real thickness of EuTe can be evaluated with an accuracy better than one monolayer. Since for EuTe layers thicker than 2 monolayers there is no difference in the character of the antiferromagnetic resonance observed for even and odd numbers of monolayers, we conclude that there is no static magnetization of the antiferromagnetic sublattices. Nevertheless, long range antiferromagnetic order is clearly evident.
\end{abstract}

PACS numbers: 75.50.Ee, 75.50.Pp, 76.50.+g

Usually it is believed that in a macroscopic, 3-dimensional antiferromagnet (AF) each magnetic sublattice is statically magnetised, but the magnetic moments compensate each other. A more sophisticated discussion of the nature of $\mathrm{AF}$, however, shows that the AF coupling leads rather to an AF correlation of the sublattices, but not necessarily to their static magnetization [1-3]. Some additional coupling, e.g., to a nuclear spin system, can lead to a symmetry breakdown and thus to the formation of static staggered magnetization. So far, there is no solid experimental evidence whether an AF is of static or of oscillatory character. In macroscopic systems the oscillation frequency tends to zero. In finite systems, the so-called macroscopic quantum coherence (MQC) rate is discussed. Some resonances, with their frequency depending on the size of the AF, were found in $A F$ nano-clusters [4], but their interpretation induced some controversy [1].

Two-dimensional AF's are intensively investigated since some classes of high temperature superconductors are 2D antiferromagnets [5]. AF correlations are well evidenced but no static staggered magnetization is proven in these materials. Moreover, there is a concept according to which coupling of conduction carriers to "oscillating" AF planes leads to the formation of superconducting pairs. Thin AF $\mathrm{MnTe}$ and MnSe layers were investigated by neutron diffraction. It was shown

*Work supported by the Committee for Scientific Research (Poland) grant No. 2P03B19108 and by the Fonds zur Förderung der Wissenschaftlichen Forschung (Austria). 
that with decreasing layer thickness a reconstruction of the $\mathrm{AF}$ structure occurs. For thin layers, a helical structure provides the compensation of the magnetic moments $[6,7]$.

In this paper, we investigate EuTe/PbTe superlattices (SL's) by means of magnetic resonance spectroscopy. The sample system differs from Mn-based SL's as no reconstruction of the AF structure occurs: (111) planes with ferromagnetically correlated $\mathrm{Eu}$ ions are antiferromagnetically correlated to the neighbouring planes [7]. Therefore, assuming static staggered magnetization, we would expect an uncompensated magnetic moment for an odd number of monolayers (ML's). Of course, the interface definition is of crucial importance here. Therefore we have studied carefully the sharpness of the real composition profile but we obtain very weak interdiffusion. The results of AF resonance (AFMR) confirm the occurrence of long range AF order and the planar structure of an AF with easy (111) magnetic planes. We find no evidence for an uncompensated magnetic moment, however, leading us to the conclusion that the ground state of thin AF EuTe should be treated as a superposition of the classical Néel states, indicating the situation of an "oscillating" AF.

We evaluate the real interdiffusion by two independent methods: (i) by analysing the magnetic anisotropy [8] and (ii) by estimating the number of isolated Eu ions which diffuse into the PbTe wells. It can be shown that in the paramagnetic regime the magnetic dipole anisotropy of the layered structure is proportional to the effective composition of the SL: $x_{\text {eff }}=\Sigma x_{i}^{2} / \Sigma x_{i}$, where $x_{i}$ is the mean composition of the $i$-th plane. Without any diffusion, we get $x_{\mathrm{eff}}=1$, and the maximum anisotropy corresponding to that of a pure EuTe layer is expected. In the other extreme of full interdiffusion a homogeneous layer of an average SL composition is formed, and magnetic anisotropy corresponds to the shape anisotropy of the diluted magnetic layer, $x_{\text {eff }}=x_{0}=\Sigma x_{i} / \Sigma i$. For intermediate cases the magnetic anisotropy varies linearly with $x_{\text {eff }}$ and allows to estimate the degree of interdiffusion.

Another test for the quality of our SL profiles is the measured amplitude of the spectra of isolated $\mathrm{Eu}$ and that of Eu-Eu pairs in the $\mathrm{PbTe}$ lattice. The discussed EPR spectra differ in character as compared to those of pure EuTe or $\mathrm{Eu}_{x} \mathrm{~Pb}_{1-x} \mathrm{Te}$. The spectrum of isolated $\mathrm{Eu}$ is observed for weak interdiffusion when there are planes with a mean composition smaller than $x=0.01$. In the investigated SL's the spectrum of the Eu impurity is easily observed before annealing if the thickness of $\mathrm{PbTe}$ layer is bigger than $10 \mathrm{ML}$. For thinner $\mathrm{PbTe}$ wells or for annealed SL's the spectrum of isolated Eu disappears, and spectra of Eu-Eu pairs and of bigger Eu clusters are observed.

The quantitative analysis of the experimental data allows to evaluate the real interdiffusion. The sharpness of profiles differs slightly from sample to sample, but generally, the interdiffusion is very weak. A substantial decrease in the Eu content occurs in the outer layers only. The effective thickness of the AF layer is by less than $1 \mathrm{ML}$ smaller than the technological thickness.

Below $T_{\mathrm{N}}$, an AFMR is observed. With a standard X-band $(\omega \approx 9 \mathrm{GHz})$ EPR spectrometer, only the mode with the resonance field, $H$, determined by the condition $\hbar \omega=g \mu_{\mathrm{B}} H \cos \Theta\left(1+\left|H_{\mathrm{A}}\right| / H_{\mathrm{E}}\right)^{1 / 2}$ can be observed [9]. Here $H_{\mathrm{A}}$ 
and $H_{\mathrm{E}}$ are the anisotropy and the exchange fields, respectively, $\mu_{\mathrm{B}}$ is the Bohr magneton and $\Theta$ is the angle between the direction of the external field and the easy plane. In Fig. 1, the angular dependence of the resonance field for several SL's is shown. The observed dependence is close to $1 / \cos \theta$, showing that only the in-plane component of the external field contributes to the AFMR. This behaviour is commonly observed for all AF samples, i.e., for samples with a nominal thickness not smaller than 3 ML's of EuTe.

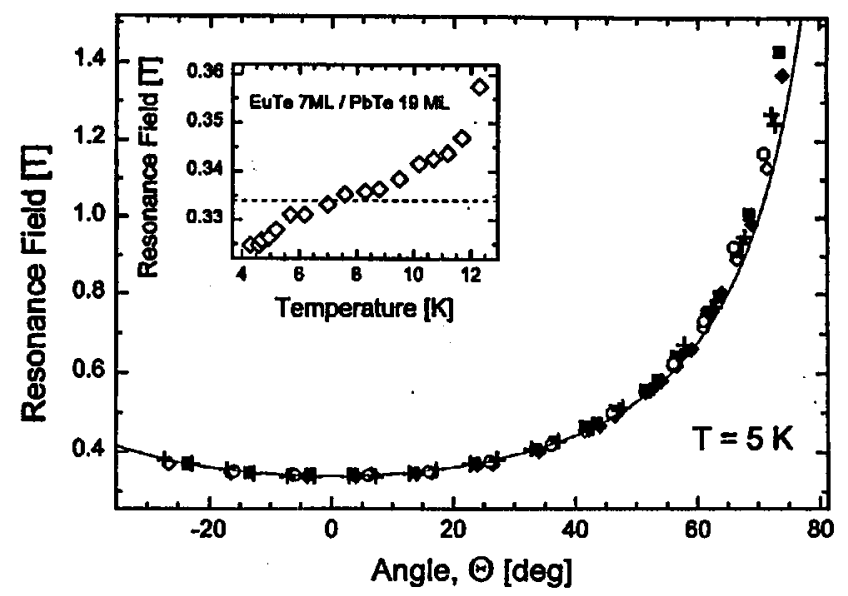

Fig. 1. Dependence of the resonance field of the AFMR for various EuTe/PbTe SL's plotted as a function of the angle from (111) plane. Thickness of EuTe layers changes from 2 to $10 \mathrm{ML}$ while thickness of PbTe spacer from 5 to $30 \mathrm{ML}$. The full line corresponds to the theoretical dependence with $H_{\mathrm{A}}=0$ and $g=2$. The inset shows an example of the temperature dependence of the resonance field for $\Theta=0$.

The inset to Fig. 1 shows the temperature dependence of the resonance field for the in-plane magnetic field direction. The character of this dependence is similar for all structures. The resonance field decreases with decreasing temperature reflecting the fact that the ratio of anisotropy and exchange fields increases with increasing correlation radius. The ratio is different from sample to sample. The increase in the resonance field at high temperatures is caused by a critical increase in the field fluctuations [8]. The observed resonance frequency at low temperature allows to estimate the anisotropy field. The shift of the resonance field from $g=2$ by about $5 \%$ shows that the ratio $H_{\mathrm{A}} / H_{\mathrm{E}}$ is about 0.1 , i.e., $H_{\mathrm{A}}$ is of the order of $1 \mathrm{~T}$ at low temperatures. This value is by an order of magnitude bigger as compared to the anisotropy observed in the paramagnetic phase. The shape anisotropy caused by the mean magnetization of the layer is similar in both phases. The much higher anisotropy in the AF phase indicates that the dipole anisotropy caused by the short range AF correlation is the dominant contribution.

The fact that the (111) growth plane is the only easy magnetic plane confirms that in the growth plane neighbours are ferromagnetically correlated and the spins in neighbouring planes are AF correlated. Since such behaviour is observed for all 
structures investigated we can conclude that there is no reconstruction of the AF structure for lower layer thickness.

We investigated about 20 different SL's with different layers thickness. The thickness of EuTe layer varied from 1 to $10 \mathrm{ML}$ and that of PbTe from 3 to $30 \mathrm{ML}$. We looked for any dependence which could distinguish layers with even and odd numbers of ML's. We investigated several quantities: the angular dependence of the resonance frequency and the resonance line width, the magnetic anisotropy, the Neel temperature, the resonance amplitude, and others. A very different, ferromagnetic-like resonance with $g_{\text {in }}=2.1$ and $g_{\text {perp }}=1.86$ is observed in a sample with a nominal thickness of $2 \mathrm{ML}$ (real thickness: $1 \mathrm{ML}$ ), suggesting a single (111) plane of EuTe with ferromagnetic correlation. But for thicker layers, which are characterised by the Néel transition, we did not find any trace of an even-odd dependence of any of the analysed parameters. The same type of AFMR was observed in all AF structures. Instead, the measured parameters change continuously with layer thickness.

We investigate SL's with very sharp composition profiles. The critical broadening of the resonance line width (both for the paramagnetic resonance and for the AFMR) proves that a long range AF order occurs. The observed AFMR shows that Eu spins are anticorrelated. The ferromagnetic-like resonance of a single ML and the kind of magnetic anisotropy of thicker AF's show that the AF structure (type II) does not change with layer thickness. Nevertheless, no trace of an uncompensated magnetic moment is evident. The results are in contrast with the classical, static Néel model of antiferromagnetism and indicate that the AF sublattices are of oscillating character. Such an AF is characterised by a vanishing magnetization of each AF sublattice.

For an uscillating AF a large staggered dynamic susceptibility is expected. Such a susceptibility may affect also the magnetic properties of conduction electrons in the semiconducting wells.

\section{References}

[1] A. Garg, Phys. Rev. Lett. 74, 1458 (1995).

[2] B. Barbara, E.M. Chudnowski, Phys. Lett. A 145, 205 (1990).

[3] R. Buczko, Z. Wilamowski, W. Jantsch, Acta Phys. Pol. A 90, 747 (1996).

[4] D.D. Awschalom, J.F. Smyth, G. Grinstein, D.P. DiVincenzo, D. Loss, Phys Rev. Lett. 68, 3092 (1992).

[5] For review see E. Dagotto, Rev. Mod. Phys. 66, 763 (1994).

[6] T.M. Giebułtowicz, N. Samarth, J.K. Furdyna, P. Kłosowski, J.J. Rhyne, Phys. Rev. B 46, 12076 (1992).

[7] T.M. Giebułtowicz, H. Luo, N. Samarth, J.K. Furdyna, V. Nunez, J.J. Rhyne, W. Faschinger, G. Springholz, G. Bauer, H. Sitter, Physica B 198, 163 (1994).

[8] Z. Wilamowski, R. Buczko, W. Jantsch, M. Ludwig, G. Springholz, Acta Phys. Pol. $A$ 90, 973 (1996).

[9] A.G. Gurevitch, G.A. Melkov, Magnitnye kolebania $i$ volny, Fizmatlit, Moskva 1994. 